\title{
On the distributive equation for $t$-representable $t$-norms generated from nilpotent and strict t-norms
}

\author{
Michał Baczyński \\ Institute of Mathematics, University of Silesia, ul. Bankowa 14, 40-007 Katowice, Poland
}

\begin{abstract}
Recently, in [4], we have discussed the following distributive equation of implications $\mathcal{I}\left(x, \mathcal{T}_{1}(y, z)\right)=$ $\mathcal{T}_{2}(\mathcal{I}(x, y), \mathcal{I}(x, z))$ over t-representable t-norms, generated from strict t-norms, in interval-valued fuzzy sets theory. In this work we continue these investigations, but with the assumption that $\mathcal{T}_{1}$ is generated from nilpotent t-norms, while $\mathcal{T}_{2}$ is generated from strict t-norms. As a byproduct result we show all solutions for the following functional equation $f\left(\min \left(u_{1}+v_{1}, a\right), \min \left(u_{2}+v_{2}, a\right)\right)=$ $f\left(u_{1}, u_{2}\right)+f\left(v_{1}, v_{2}\right)$ related to this case.
\end{abstract}

Keywords: Interval-valued fuzzy sets, intuitionistic fuzzy sets, fuzzy implication, triangular norm, distributivity equations, functional equations.

\section{Introduction}

Distributivity of fuzzy implications over different fuzzy logic connectives has been studied in the recent past by many authors (see [2], [26], [7], [23], $[24],[6],[3])$. These equations have a very important role to play in efficient inferencing in approximate reasoning, especially in fuzzy control systems. Since all the rules of an inference engine are exercised during every inference cycle, the number of rules directly affects the computational duration of the overall application. To reduce the complexity of fuzzy "IF-THEN" rules, Combs and Andrews [9] required of the following classical tautology

$$
(p \wedge q) \rightarrow r=(p \rightarrow r) \vee(q \rightarrow r)
$$

Subsequently, there were many discussions (see [10], [11], [16], [22]), most of them pointed out the need for a theoretical investigation required for employing such equations, as concluded by Dick and Kandel [16], "Future work on this issue will require an examination of the properties of various combinations of fuzzy unions, intersections and implications". An overview of the most important methods that reduce the complexity of different inference systems can be found in [5, Chapter 8].

Recently, in [4], we have discussed the distributive equation of implications

$$
\mathcal{I}\left(x, \mathcal{T}_{1}(y, z)\right)=\mathcal{T}_{2}(\mathcal{I}(x, y), \mathcal{I}(x, z))
$$

over t-representable t-norms, generated from strict t-norms, in interval-valued fuzzy sets theory. In this work we continue these investigations, but with the assumption that $\mathcal{T}_{1}$ is generated from nilpotent t-norms, while $\mathcal{T}_{2}$ is generated from strict t-norms. In [4], as a byproduct result, we have obtained the solutions of the following functional equation:

$$
f\left(u_{1}+v_{1}, u_{2}+v_{2}\right)=f\left(u_{1}, u_{2}\right)+f\left(v_{1}, v_{2}\right),
$$

satisfied for all $\left(u_{1}, u_{2}\right),\left(v_{1}, v_{2}\right) \in L^{\infty}$, where $L^{\infty}=$ $\left\{\left(u_{1}, u_{2}\right) \in[0, \infty]^{2} \mid u_{1} \geq u_{2}\right\}$ and $f: L^{\infty} \rightarrow[0, \infty]$ is an unknown function. In this article we will present all solutions of the following equation:

$$
\begin{aligned}
& f\left(\min \left(u_{1}+v_{1}, a\right),\right.\left.\min \left(u_{2}+v_{2}, a\right)\right) \\
&=f\left(u_{1}, u_{2}\right)+f\left(v_{1}, v_{2}\right),
\end{aligned}
$$

satisfied for all $\left(u_{1}, u_{2}\right),\left(v_{1}, v_{2}\right) \in L^{a}$, where $a>0$ is fixed real number, $f: L^{a} \rightarrow[0, \infty]$ is an unknown function and $L^{a}=\left\{\left(u_{1}, u_{2}\right) \in[0, a]^{2} \mid u_{1} \geq u_{2}\right\}$. This equation is related to the case with nilpotent and strict t-norms. Such theoretical developments connected with solutions of different functional equations can be also useful in other topics like fuzzy mathematical morphology (see [12]) or similarity measures (cf. [8]).

\section{Intuitionistic and interval-valued fuzzy sets theories}

Intuitionistic fuzzy sets theory introduced by Atanassov [1] assign to each element of the universe not only a membership degree, but also a nonmembership degree (for the discussion connected with the proposed terminology see [17]).

Definition 2.1. An intuitionistic fuzzy set $A$ on $X$ is a set

$$
A=\left\{\left(x, \mu_{A}(x), \nu_{A}(x)\right): x \in X\right\},
$$

where $\mu_{A}, \nu_{A}: X \rightarrow[0,1]$ are called, respectively, the membership function and the non-membership function. Moreover they satisfy the condition

$$
\mu_{A}(x)+\nu_{A}(x) \leq 1, \quad x \in X .
$$

Let us define

$$
\begin{gathered}
L^{*}=\left\{\left(x_{1}, x_{2}\right) \in[0,1]^{2}: x_{1}+x_{2} \leq 1\right\}, \\
\left(x_{1}, x_{2}\right) \leq_{L^{*}}\left(y_{1}, y_{2}\right) \Longleftrightarrow x_{1} \leq y_{1} \wedge x_{2} \geq y_{2} .
\end{gathered}
$$


One can easily observe that $\mathcal{L}^{*}=\left(L^{*}, \leq_{L^{*}}\right)$ is a complete lattice with units $0_{L^{*}}=(0,1)$ and $1_{L^{*}}=$ $(1,0)$. Moreover, an intuitionistic fuzzy set $A$ on $X$ can be represented by the $\mathcal{L}^{*}$-fuzzy set given by $A: X \rightarrow L^{*}$.

Another extension of fuzzy sets theory is intervalvalued fuzzy sets theory introduced, independently, by Sambuc [25] and Gorzałczany [19], in which to each element of the universe a closed subinterval of the unit interval is assigned - it can be used as an approximation of the unknown membership degree. Let us define

$$
\begin{gathered}
L^{I}=\left\{\left(x_{1}, x_{2}\right) \in[0,1]^{2}: x_{1} \leq x_{2}\right\}, \\
\left(x_{1}, x_{2}\right) \leq_{L^{I}}\left(y_{1}, y_{2}\right) \Longleftrightarrow x_{1} \leq y_{1} \wedge x_{2} \leq y_{2} .
\end{gathered}
$$

In the sequel, if $x \in L^{I}$, then we denote it by $x=$ $\left[x_{1}, x_{2}\right]$. One can easily observe that $\mathcal{L}^{I}=\left(L^{I}, \leq_{L^{I}}\right)$ is also a complete lattice with units $0_{\mathcal{L}^{I}}=[0,0]$ and $1_{\mathcal{L}^{I}}=[1,1]$.

Definition 2.2. An interval-valued fuzzy set on $X$ is a mapping $A: X \rightarrow L^{I}$.

It is important to notice that in [13] it is shown that intuitionistic fuzzy sets theory is equivalent, from the mathematical point of view, to intervalvalued fuzzy sets theory. In fact, we can see the point $\left(x_{1}, x_{2}\right) \in L^{*}$ as the interval $\left[x_{1}, 1-x_{2}\right] \in L^{I}$ (and vice-verse). Since we are limited in number of pages, in this article we will discuss main results in the language of interval-valued fuzzy sets, but they can be easily transformed to the intuitionistic case.

\section{Basic fuzzy connectives}

We assume that the reader is familiar with the classical results concerning basic fuzzy logic connectives, but we briefly mention some of the results employed in the rest of the work.

Definition 3.1. Let $\mathcal{L}=\left(L, \leq_{L}\right)$ be a complete lattice. An associative, commutative operation $\mathcal{T}: L^{2} \rightarrow L$ is called a t-norm if it is increasing and $1_{\mathcal{L}}$ is the neutral element of $\mathcal{T}$.

Definition 3.2. A t-norm $T$ on $([0,1], \leq)$ is said to be nilpotent, if it is continuous and if each $x \in(0,1)$ is a nilpotent element of $T$, i.e., if there exists $n \in \mathbb{N}$ such that $x_{T}^{[n]}=0$, where

$$
x_{T}^{[n]}:= \begin{cases}x, & \text { if } n=1 \\ T\left(x, x_{T}^{[n-1]}\right), & \text { if } n>1 .\end{cases}
$$

Definition 3.3. A t-norm $T$ on $([0,1], \leq)$ is said to be strict, if it is continuous and strictly monotone, i.e., $T(x, y)<T(x, z)$ whenever $x>0$ and $y<z$.

The following characterizations of nilpotent and strict t-norms are well-known in the literature.
Theorem 3.4 ([20]). A function $T:[0,1]^{2} \rightarrow[0,1]$ is a nilpotent t-norm if and only if there exists a continuous, strictly decreasing function $t:[0,1] \rightarrow$ $[0, \infty)$ with $t(1)=0$, which is uniquely determined up to a positive multiplicative constant, such that

$T(x, y)=t^{-1}(\min (t(x)+t(y), t(0))), \quad x, y \in[0,1]$.

Theorem 3.5 ([20]). A function $T:[0,1]^{2} \rightarrow[0,1]$ is a strict $t$-norm if and only if there exists a continuous, strictly decreasing function $t:[0,1] \rightarrow[0, \infty]$ with $t(1)=0$ and $t(0)=\infty$, which is uniquely determined up to a positive multiplicative constant, such that

$$
T(x, y)=t^{-1}(t(x)+t(y)), \quad x, y \in[0,1] .
$$

In our article we shall consider the following special class of t-norms.

Definition 3.6 (see [14]). A t-norm $\mathcal{T}$ on $\mathcal{L}^{I}$ is called t-representable if there exist t-norms $T_{1}$ and $T_{2}$ on $([0,1], \leq)$ such that $T_{1} \leq T_{2}$ and

$$
\mathcal{T}\left(\left[x_{1}, x_{2}\right],\left[y_{1}, y_{2}\right]\right)=\left[T_{1}\left(x_{1}, y_{1}\right), T_{2}\left(x_{2}, y_{2}\right)\right],
$$

for all $\left[x_{1}, x_{2}\right],\left[y_{1}, y_{2}\right] \in L^{I}$.

It should be noted that not all t-norms on $\mathcal{L}^{I}$ are t-representable (see [14]).

One possible definition of an implication on $\mathcal{L}^{I}$ is based on the well-accepted notation introduced by Fodor and Roubens [18] (see also [5], [15] and [21]).

Definition 3.7. Let $\mathcal{L}=\left(L, \leq_{L}\right)$ be a complete lattice. A function $\mathcal{I}: L^{2} \rightarrow \bar{L}$ is called a fuzzy implication on $\mathcal{L}$ if it is decreasing with respect to the first variable, increasing with respect to the second variable and fulfills the following conditions: $\mathcal{I}\left(0_{\mathcal{L}}, 0_{\mathcal{L}}\right)=\mathcal{I}\left(1_{\mathcal{L}}, 1_{\mathcal{L}}\right)=\mathcal{I}\left(0_{\mathcal{L}}, 1_{\mathcal{L}}\right)=1_{\mathcal{L}}$ and $\mathcal{I}\left(1_{\mathcal{L}}, 0_{\mathcal{L}}\right)=0_{\mathcal{L}}$.

\section{Some new results pertaining to functional equations}

In this section we show one new result related to functional equations, which will be crucial in obtaining main results.

Proposition 4.1 ([3, Proposition 3.6]). Fix real $a>0$. For a function $f:[0, a] \rightarrow[0, \infty]$ the following statements are equivalent:

(i) $f$ satisfies the functional equation

$$
f(\min (x+y, a))=f(x)+f(y),
$$

for all $x, y \in[0, a]$.

(ii) Either $f=0$, or $f=\infty$, or

$$
f(x)=\left\{\begin{array}{ll}
0, & \text { if } x=0, \\
\infty, & \text { if } x>0,
\end{array} \quad \text { for all } x \in[0, a]\right.
$$


Proposition 4.2. Fix real $a>0$. Let $L^{a}=$ $\left\{\left(u_{1}, u_{2}\right) \in[0, a]^{2}: u_{1} \geq u_{2}\right\}$. For a function $f: L^{a} \rightarrow[0, \infty]$ the following statements are equivalent:

(i) $f$ satisfies the functional equation

$$
\begin{aligned}
& f\left(\min \left(u_{1}+v_{1}, a\right),\right.\left.\min \left(u_{2}+v_{2}, a\right)\right) \\
&=f\left(u_{1}, u_{2}\right)+f\left(v_{1}, v_{2}\right),
\end{aligned}
$$

for all $\left(u_{1}, u_{2}\right),\left(v_{1}, v_{2}\right) \in L^{a}$.

(ii) Either

$$
f=0
$$

or

$$
f=\infty
$$

or

$$
f\left(u_{1}, u_{2}\right)= \begin{cases}0, & \text { if } u_{2}=0 \\ \infty, & \text { if } u_{2}>0\end{cases}
$$

or

$$
f\left(u_{1}, u_{2}\right)= \begin{cases}0, & \text { if } u_{1}=0 \\ \infty, & \text { if } u_{1}>0\end{cases}
$$

for all $\left(u_{1}, u_{2}\right) \in L^{a}$.

Proof. $($ ii $) \Longrightarrow($ i $)$ It is a direct calculation that all the above functions satisfy (A).

$(i) \Longrightarrow($ ii $)$ Let a function $f: L^{a} \rightarrow[0, \infty]$ satisfy equation $(\mathrm{A})$ for all $\left(u_{1}, u_{2}\right),\left(v_{1}, v_{2}\right) \in L^{a}$. Setting $u_{1}=v_{1}=a$ in $(\mathrm{A})$ we get

$f\left(\min (a+a, a), \min \left(u_{2}+v_{2}, a\right)\right)=f\left(a, u_{2}\right)+f\left(a, v_{2}\right)$,

for all $u_{2}, v_{2} \in[0, a]$. Let us denote $f_{a}(x):=f(a, x)$, for $x \in[0, a]$. Therefore, we get

$$
f_{a}\left(\min \left(u_{2}+v_{2}, a\right)\right)=f_{a}\left(u_{2}\right)+f_{a}\left(v_{2}\right),
$$

for all $u_{2}, v_{2} \in[0, a]$. For this equation we can use solutions from Proposition 4.1. We have 3 possible cases for the function $f_{a}$.

1. If $f_{a}=0$, then putting $u_{1}=u_{2}=a$ in (A) we have

$$
\begin{aligned}
& f\left(\min \left(a+v_{1}, a\right),\right.\left.\min \left(a+v_{2}, a\right)\right) \\
&=f(a, a)+f\left(v_{1}, v_{2}\right),
\end{aligned}
$$

for all $\left(v_{1}, v_{2}\right) \in L^{a}$, so

$$
f(a, a)=f(a, a)+f\left(v_{1}, v_{2}\right),
$$

thus $0=f\left(v_{1}, v_{2}\right)$ for all $\left(v_{1}, v_{2}\right) \in L^{a}$ and we get first solution $f=0$, i.e., (S1).

2. If $f_{a}(x)=\left\{\begin{array}{ll}0, & \text { if } x=0 \\ \infty, & \text { if } x>0\end{array}\right.$, then putting $u_{1}=a$ in $(\mathrm{A})$ we have

$$
f\left(a, \min \left(u_{2}+v_{2}, a\right)\right)=f\left(a, u_{2}\right)+f\left(v_{1}, v_{2}\right),
$$

for all $\left(v_{1}, v_{2}\right) \in L^{a}$. If we take $u_{2}=v_{2}=0$ above, then we get

$$
f(a, 0)=f(a, 0)+f\left(v_{1}, 0\right), \quad v_{1} \in[0, a],
$$

thus $f\left(v_{1}, 0\right)=0$ for all $v_{1} \in[0, a]$.

If we take $u_{2}=0$ and $v_{2}>0$ above, then we get

$$
f\left(a, v_{2}\right)=f(a, 0)+f\left(v_{1}, v_{2}\right),
$$

for all $v_{1} \in[0, a]$, therefore $\infty=0+f\left(v_{1}, v_{2}\right)$, i.e., $f\left(v_{1}, v_{2}\right)=\infty$. In summary, we get the solution (S3).

Therefore, we need to solve our equation with the last possible assumption that $f_{a}=\infty$. Setting now $u_{2}=v_{2}=0$ in $(\mathrm{A})$ we get

$$
f\left(\min \left(u_{1}+v_{1}, a\right), 0\right)=f\left(u_{1}, 0\right)+f\left(v_{1}, 0\right),
$$

where $u_{1}, v_{1} \in[0, a]$. Let us denote $f^{0}(x):=f(x, 0)$, for all $x \in[0, a]$. Hence, we obtain the following functional equation

$$
f^{0}\left(\min \left(u_{1}+v_{1}, a\right)\right)=f^{0}\left(u_{1}\right)+f^{0}\left(v_{1}\right),
$$

satisfied for all $u_{1}, v_{1} \in[0, a]$. For this equation we again can use solutions described in Proposition 4.1. We have 3 possible cases for the function $f^{0}$.

1. If $f^{0}=0$, then $f(a, 0)=0$, which contradicts our assumption $f_{a}=\infty$.

2. If $f^{0}=\infty$, then putting $u_{1}=u_{2}=0$ in (A) we have

$$
f\left(v_{1}, v_{2}\right)=f(0,0)+f\left(v_{1}, v_{2}\right),
$$

for all $\left(v_{1}, v_{2}\right) \in L^{a}$, thus $f\left(v_{1}, v_{2}\right)=\infty$, hence we get next possible solution $f=\infty$, i.e., (S2).

3. If $f^{0}(x)=\left\{\begin{array}{ll}0, & \text { if } x=0 \\ \infty, & \text { if } x>0\end{array}\right.$, then putting $u_{2}=0$ in $(\mathrm{A})$ we have

$$
f\left(\min \left(u_{1}+v_{1}, a\right), v_{2}\right)=f\left(u_{1}, 0\right)+f\left(v_{1}, v_{2}\right) .
$$

Let us assume that $u_{1}>0$ and $v_{1}=v_{2}$ above. Then we get

$$
f\left(\min \left(u_{1}+v_{2}, a\right), v_{2}\right)=\infty+f\left(v_{2}, v_{2}\right),
$$

hence

$$
f\left(\min \left(u_{1}+v_{2}, a\right), v_{2}\right)=\infty,
$$

for all $u_{1} \in(0, a], v_{2} \in[0, a]$. Observe that $\min \left(u_{1}+v_{2}, a\right) \in\left(v_{2}, a\right]$ and $v_{2} \in[0, a]$, thus we have obtained the result that $f\left(x_{1}, x_{2}\right)=\infty$ for any $\left(x_{1}, x_{2}\right) \in L^{a}$ such that $x_{1}>x_{2}$.

Let us take now $u_{2}=u_{1}$ and $v_{2}=v_{1}$ in (A). Then we have

$$
\begin{aligned}
& f\left(\min \left(u_{1}+v_{1}, a\right),\right.\left.\min \left(u_{1}+v_{1}, a\right)\right) \\
&=f\left(u_{1}, u_{1}\right)+f\left(v_{1}, v_{1}\right),
\end{aligned}
$$

for all $u_{1}, v_{1} \in[0, a]$. Let us denote by $g(x):=$ $f(x, x)$, for $x \in[0, a]$. Therefore we obtain the following functional equation

$$
g\left(\min \left(u_{1}+v_{1}, a\right)\right)=g\left(u_{1}\right)+g\left(v_{1}\right),
$$

satisfied for all $u_{1}, v_{1} \in[0, a]$. For this equation we again can use solutions described in Proposition 4.1. We have 3 possible cases for the function $g$. 
(a) If $g=0$, then $f(a, a)=0$, which contradicts our assumption $f_{a}=\infty$.

(b) If $g=\infty$, then $f(0,0)=\infty$, which contradicts our assumption 3 . on function $f^{0}$.

(c) If $g(x)=\left\{\begin{array}{ll}0, & \text { if } x=0 \\ \infty, & \text { if } x>0\end{array}\right.$, then taking into account previous calculations we get the solution ( $\mathrm{S} 4)$ in this case.

\section{Distributive equation for t-representable t-norms}

In this section we will show how we can use solutions presented in Proposition 4.2 to obtain all solutions, in particular fuzzy implications, of our main distributive equation

$$
\mathcal{I}\left(x, \mathcal{T}_{1}(y, z)\right)=\mathcal{T}_{2}(\mathcal{I}(x, y), \mathcal{I}(x, z)),
$$

satisfied for all $x, y, z \in L^{I}$, where $\mathcal{I}$ is an unknown function, $\mathcal{T}_{1}$ is a t-representable t-norm on $\mathcal{L}^{I}$ generated from nilpotent t-norms $T_{1}, T_{2}$ and $\mathcal{T}_{2}$ is a t-representable t-norm on $\mathcal{L}^{I}$ generated from strict t-norms $T_{3}, T_{4}$.

Assume that projection mappings on $\mathcal{L}^{I}$ are defined as the following:

$$
\operatorname{pr}_{1}\left(\left[x_{1}, x_{2}\right]\right)=x_{1}, \quad \operatorname{pr}_{2}\left(\left[x_{1}, x_{2}\right]\right)=x_{2},
$$

for $\left[x_{1}, x_{2}\right] \in L^{I}$. In [4] we have shown that if $\mathcal{T}_{1}$ and $\mathcal{T}_{2}$ on $\mathcal{L}^{I}$ are t-representable, then

$$
\begin{aligned}
g_{\left[x_{1}, x_{2}\right]}^{1} & \left(\left[T_{1}\left(y_{1}, z_{1}\right), T_{2}\left(y_{2}, z_{2}\right)\right]\right) \\
& =T_{3}\left(g_{\left[x_{1}, x_{2}\right]}^{1}\left(\left[y_{1}, y_{2}\right]\right), g_{\left[x_{1}, x_{2}\right]}^{1}\left(\left[z_{1}, z_{2}\right]\right)\right), \\
g_{\left(x_{1}, x_{2}\right)}^{2} & \left(\left[T_{1}\left(y_{1}, z_{1}\right), T_{2}\left(y_{2}, z_{2}\right)\right]\right) \\
& =T_{4}\left(g_{\left[x_{1}, x_{2}\right]}^{2}\left(\left[y_{1}, y_{2}\right]\right), g_{\left[x_{1}, x_{2}\right]}^{2}\left(\left[z_{1}, z_{2}\right]\right)\right),
\end{aligned}
$$

where $\left[x_{1}, x_{2}\right] \in L^{I}$ is arbitrarily fixed and functions $g_{\left[x_{1}, x_{2}\right]}^{1}, g_{\left[x_{1}, x_{2}\right]}^{2}: L^{I} \rightarrow L^{I}$ are defined by

$$
\begin{aligned}
& g_{\left[x_{1}, x_{2}\right]}^{1}(\cdot):=p r_{1} \circ \mathcal{I}\left(\left[x_{1}, x_{2}\right], \cdot\right), \\
& g_{\left[x_{1}, x_{2}\right]}^{2}(\cdot):=p r_{2} \circ \mathcal{I}\left(\left[x_{1}, x_{2}\right], \cdot\right) .
\end{aligned}
$$

Let us assume that $T_{1}=T_{2}$ is a nilpotent t-norm generated from additive generator $t_{1}$ and $T_{3}=T_{4}$ is a strict t-norm generated from additive generator $t_{3}$. Using the representations of nilpotent t-norms (Theorem 3.4) and strict t-norms (Theorem 3.5) we can transform our problem to the following equation (for a simplicity we deal only with $g^{1}$ now):

$$
\begin{gathered}
g_{\left[x_{1}, x_{2}\right]}^{1}\left(\left[t_{1}^{-1}\left(\min \left(t_{1}\left(y_{1}\right)+t_{1}\left(z_{1}\right), t_{1}(0)\right)\right),\right.\right. \\
\left.\left.t_{1}^{-1}\left(\min \left(t_{1}\left(y_{2}\right)+t_{1}\left(z_{2}\right), t_{1}(0)\right)\right)\right]\right) \\
=t_{3}^{-1}\left(t_{3}\left(g_{\left[x_{1}, x_{2}\right]}^{1}\left(\left[y_{1}, y_{2}\right]\right)\right)\right. \\
\left.+t_{3}\left(g_{\left[x_{1}, x_{2}\right]}^{1}\left(\left[z_{1}, z_{2}\right]\right)\right)\right) .
\end{gathered}
$$

Hence

$$
\begin{gathered}
t_{3} \circ g_{\left[x_{1}, x_{2}\right]}^{1}\left(\left[t_{1}^{-1}\left(\min \left(t_{1}\left(y_{1}\right)+t_{1}\left(z_{1}\right), t_{1}(0)\right)\right),\right.\right. \\
\left.\left.t_{1}^{-1}\left(\min \left(t_{1}\left(y_{2}\right)+t_{1}\left(z_{2}\right), t_{1}(0)\right)\right)\right]\right) \\
=t_{3} \circ g_{\left[x_{1}, x_{2}\right]}^{1}\left(\left[y_{1}, y_{2}\right]\right) \\
\quad+t_{3} \circ g_{\left[x_{1}, x_{2}\right]}^{1}\left(\left[z_{1}, z_{2}\right]\right) .
\end{gathered}
$$

Let us put $t_{1}\left(y_{1}\right)=u_{1}, t_{1}\left(y_{2}\right)=u_{2}, t_{1}\left(z_{1}\right)=v_{1}$ and $t_{1}\left(z_{2}\right)=v_{2}$. Of course $u_{1}, u_{2}, v_{1}, v_{2} \in\left[0, t_{1}(0)\right]$. Moreover $\left[y_{1}, y_{2}\right],\left[z_{1}, z_{2}\right] \in L^{I}$, thus $y_{1} \leq y_{2}$ and $z_{1} \leq z_{2}$. The generator $t_{1}$ is strictly decreasing, so $u_{1} \geq u_{2}$ and $v_{1} \geq v_{2}$. If we put

$f_{\left[x_{1}, x_{2}\right]}(u, v):=t_{3} \circ p r_{1} \circ \mathcal{I}\left(\left[x_{1}, x_{2}\right],\left[t_{1}^{-1}(u), t_{1}^{-1}(v)\right]\right)$,

where $u, v \in\left[0, t_{1}(0)\right], u \geq v$, then we get the following functional equation

$$
\begin{gathered}
f_{\left[x_{1}, x_{2}\right]}\left(\min \left(u_{1}+v_{1}, t_{1}(0)\right), \min \left(u_{2}+v_{2}, t_{1}(0)\right)\right) \\
=f_{\left[x_{1}, x_{2}\right]}\left(u_{1}, u_{2}\right)+f_{\left[x_{1}, x_{2}\right]}\left(v_{1}, v_{2}\right)
\end{gathered}
$$

satisfied for all $\left(u_{1}, u_{2}\right),\left(v_{1}, v_{2}\right) \in L^{t_{1}(0)}$. Of course function $f_{\left[x_{1}, x_{2}\right]}: L^{t_{1}(0)} \rightarrow[0, \infty]$ is unknown above. In a same way we can repeat all the above calculations, but for the function $g^{2}$, to obtain the following functional equation

$$
\begin{gathered}
f^{\left[x_{1}, x_{2}\right]}\left(\min \left(u_{1}+v_{1}, t_{1}(0)\right), \min \left(u_{2}+v_{2}, t_{1}(0)\right)\right) \\
=f^{\left[x_{1}, x_{2}\right]}\left(u_{1}, u_{2}\right)+f^{\left[x_{1}, x_{2}\right]}\left(v_{1}, v_{2}\right)
\end{gathered}
$$

satisfied for all $\left(u_{1}, u_{2}\right),\left(v_{1}, v_{2}\right) \in L^{t_{1}(0)}$, where

$f^{\left[x_{1}, x_{2}\right]}(u, v):=t_{3} \circ p r_{2} \circ \mathcal{I}\left(\left[x_{1}, x_{2}\right],\left[t_{1}^{-1}(u), t_{1}^{-1}(v)\right]\right)$

is an unknown function. Observe that (1) and (2) are exactly our functional equation (A). Therefore, using solutions of Proposition 4.2, we are able to obtain the description of the vertical section $\mathcal{I}\left(\left[x_{1}, x_{2}\right], \cdot\right)$ for a fixed $\left[x_{1}, x_{2}\right] \in L^{I}$. Since in this proposition we have 4 possible solutions, we should have 16 different solutions of (D1). Observe now that some of these solutions are not good, since the range of $\mathcal{I}$ is $L^{I}$. Now, we will check all possibilities. Let us fix arbitrarily $\left[x_{1}, x_{2}\right] \in L^{I}$ and consider 16 different cases:

1. $f_{\left[x_{1}, x_{2}\right]}=0$ and $f^{\left[x_{1}, x_{2}\right]}=0$.

This implies that

$$
t_{3} \circ p r_{1} \circ \mathcal{I}\left(\left[x_{1}, x_{2}\right],\left[t_{1}^{-1}\left(u_{1}\right), t_{1}^{-1}\left(u_{2}\right)\right]\right)=0,
$$

for all $u_{1}, u_{2} \in\left[0, t_{1}(0)\right], u_{1} \geq u_{2}$, thus

$$
p r_{1} \circ \mathcal{I}\left(\left[x_{1}, x_{2}\right],\left[y_{1}, y_{2}\right]\right)=1, \quad\left[y_{1}, y_{2}\right] \in L^{I}
$$

Similarly we get

$$
p r_{2} \circ \mathcal{I}\left(\left[x_{1}, x_{2}\right],\left[y_{1}, y_{2}\right]\right)=1, \quad\left[y_{1}, y_{2}\right] \in L^{I}
$$

In summary, we obtain the following correct solution:

$$
\mathcal{I}\left(\left[x_{1}, x_{2}\right],\left[y_{1}, y_{2}\right]\right)=[1,1]=1_{\mathcal{L}^{I}}
$$


2. $f_{\left[x_{1}, x_{2}\right]}=0$ and $f^{\left[x_{1}, x_{2}\right]}=\infty$.

This implies that

$p r_{1} \circ \mathcal{I}\left(\left[x_{1}, x_{2}\right],\left[y_{1}, y_{2}\right]\right)=1, \quad\left[y_{1}, y_{2}\right] \in L^{I}$,

while

$p r_{2} \circ \mathcal{I}\left(\left[x_{1}, x_{2}\right],\left[y_{1}, y_{2}\right]\right)=0, \quad\left[y_{1}, y_{2}\right] \in L^{I}$.

In summary we get the following function

$$
\mathcal{I}\left(\left[x_{1}, x_{2}\right],\left[y_{1}, y_{2}\right]\right)=[1,0],
$$

but this solution is not correct, since $[1,0] \notin L^{I}$.

3. $f_{\left[x_{1}, x_{2}\right]}=0$ and $f^{\left[x_{1}, x_{2}\right]}\left(u_{1}, u_{2}\right)=$ $\begin{cases}0, & \text { if } u_{2}=0 \\ \infty, & \text { if } u_{2}>0 .\end{cases}$

Similarly as above one can check that such situation does not give the correct solution.

4. $f_{\left[x_{1}, x_{2}\right]}=0$ and $f^{\left[x_{1}, x_{2}\right]}\left(u_{1}, u_{2}\right)=$ $\begin{cases}0, & \text { if } u_{1}=0, \\ \infty, & \text { if } u_{1}>0 .\end{cases}$

One can check that such situation does not give the correct solution.

5. $f_{\left[x_{1}, x_{2}\right]}=\infty$ and $f^{\left[x_{1}, x_{2}\right]}=0$.

This implies that

$p r_{1} \circ \mathcal{I}\left(\left[x_{1}, x_{2}\right],\left[y_{1}, y_{2}\right]\right)=0, \quad\left[y_{1}, y_{2}\right] \in L^{I}$,

while

$p r_{2} \circ \mathcal{I}\left(\left[x_{1}, x_{2}\right],\left[y_{1}, y_{2}\right]\right)=1, \quad\left[y_{1}, y_{2}\right] \in L^{I}$.

In summary we obtain the following correct solution:

$$
\mathcal{I}\left(\left[x_{1}, x_{2}\right],\left[y_{1}, y_{2}\right]\right)=[0,1] .
$$

6. $f_{\left[x_{1}, x_{2}\right]}=\infty$ and $f^{\left[x_{1}, x_{2}\right]}=\infty$.

In this case we obtain the following correct solution:

$$
\mathcal{I}\left(\left[x_{1}, x_{2}\right],\left[y_{1}, y_{2}\right]\right)=[0,0]=0_{\mathcal{L}^{I}} .
$$

7. $f_{\left[x_{1}, x_{2}\right]}=\infty$ and $f^{\left[x_{1}, x_{2}\right]}\left(u_{1}, u_{2}\right)=$ $\begin{cases}0, & \text { if } u_{2}=0 \\ \infty, & \text { if } u_{2}>0 .\end{cases}$

In this case we obtain the following correct solution:

$$
\mathcal{I}\left(\left[x_{1}, x_{2}\right],\left[y_{1}, y_{2}\right]\right)= \begin{cases}{[0,1],} & \text { if } y_{2}=1 \\ {[0,0],} & \text { if } y_{2}<1\end{cases}
$$

8. $f_{\left[x_{1}, x_{2}\right]}=\infty$ and $f^{\left[x_{1}, x_{2}\right]}\left(u_{1}, u_{2}\right)=$ $\begin{cases}0, & \text { if } u_{1}=0 \\ \infty, & \text { if } u_{1}>0 .\end{cases}$

In this case we obtain the following correct solution:

$$
\mathcal{I}\left(\left[x_{1}, x_{2}\right],\left[y_{1}, y_{2}\right]\right)= \begin{cases}{[0,1],} & \text { if } y_{1}=1 \\ {[0,0],} & \text { if } y_{1}<1\end{cases}
$$

9. $f_{\left[x_{1}, x_{2}\right]}\left(u_{1}, u_{2}\right)=\left\{\begin{array}{ll}0, & \text { if } u_{2}=0, \\ \infty, & \text { if } u_{2}>0 .\end{array}\right.$ and $f^{\left[x_{1}, x_{2}\right]}=0$.

In this case we obtain the following correct solution:

$$
\mathcal{I}\left(\left[x_{1}, x_{2}\right],\left[y_{1}, y_{2}\right]\right)= \begin{cases}{[1,1],} & \text { if } y_{2}=1, \\ {[0,1],} & \text { if } y_{2}<1 .\end{cases}
$$

10. $f_{\left[x_{1}, x_{2}\right]}\left(u_{1}, u_{2}\right)=\left\{\begin{array}{ll}0, & \text { if } u_{2}=0, \\ \infty, & \text { if } u_{2}>0 .\end{array}\right.$ and $f^{\left[x_{1}, x_{2}\right]}=\infty$.

One can check that such situation does not give the correct solution.

11. $f_{\left[x_{1}, x_{2}\right]}\left(u_{1}, u_{2}\right)=\left\{\begin{array}{ll}0, & \text { if } u_{2}=0, \\ \infty, & \text { if } u_{2}>0 .\end{array}\right.$ and $f^{\left[x_{1}, x_{2}\right]}\left(u_{1}, u_{2}\right)=\left\{\begin{array}{ll}0, & \text { if } u_{2}=0 \\ \infty, & \text { if } u_{2}>0 .\end{array}\right.$.

In this case we obtain the following correct solution:

$$
\mathcal{I}\left(\left[x_{1}, x_{2}\right],\left[y_{1}, y_{2}\right]\right)= \begin{cases}{[1,1],} & \text { if } y_{2}=1, \\ {[0,0],} & \text { if } y_{2}<1 .\end{cases}
$$

12. $f_{\left[x_{1}, x_{2}\right]}\left(u_{1}, u_{2}\right)=\left\{\begin{array}{ll}0, & \text { if } u_{2}=0, \\ \infty, & \text { if } u_{2}>0 .\end{array}\right.$ and $f^{\left[x_{1}, x_{2}\right]}\left(u_{1}, u_{2}\right)=\left\{\begin{array}{ll}0, & \text { if } u_{1}=0, \\ \infty, & \text { if } u_{1}>0 .\end{array}\right.$.

One can check that such situation does not give the correct solution. Indeed, when $\left[y_{1}, y_{2}\right]=$ $[0.5,1]$, then we get

$$
\mathcal{I}\left(\left[x_{1}, x_{2}\right],[0.5,1]\right)=[1,0] .
$$

13. $f_{\left[x_{1}, x_{2}\right]}\left(u_{1}, u_{2}\right)=\left\{\begin{array}{ll}0, & \text { if } u_{1}=0, \\ \infty, & \text { if } u_{1}>0 .\end{array}\right.$ and $f^{\left[x_{1}, x_{2}\right]}=0$.

In this case we obtain the following correct solution:

$$
\mathcal{I}\left(\left[x_{1}, x_{2}\right],\left[y_{1}, y_{2}\right]\right)= \begin{cases}{[1,1],} & \text { if } y_{1}=1 \\ {[0,1],} & \text { if } y_{1}<1 .\end{cases}
$$

14. $f_{\left[x_{1}, x_{2}\right]}\left(u_{1}, u_{2}\right)=\left\{\begin{array}{ll}0, & \text { if } u_{1}=0, \\ \infty, & \text { if } u_{1}>0,\end{array}\right.$ and $f^{\left[x_{1}, x_{2}\right]}=\infty$.

One can check that such situation does not give the correct solution.

15. $f_{\left[x_{1}, x_{2}\right]}\left(u_{1}, u_{2}\right)=\left\{\begin{array}{ll}0, & \text { if } u_{1}=0, \\ \infty, & \text { if } u_{1}>0 .\end{array}\right.$ and $f^{\left[x_{1}, x_{2}\right]}\left(u_{1}, u_{2}\right)= \begin{cases}0, & \text { if } u_{2}=0, \\ \infty, & \text { if } u_{2}>0 .\end{cases}$

In this case we obtain the following correct solution:

$\mathcal{I}\left(\left[x_{1}, x_{2}\right],\left[y_{1}, y_{2}\right]\right)= \begin{cases}{[1,1],} & \text { if } y_{1}=1, \\ {[0,1],} & \text { if } y_{1}<1 \& y_{2}=1, \\ {[0,0],} & \text { if } y_{2}<1 .\end{cases}$ 
16. $f_{\left[x_{1}, x_{2}\right]}\left(u_{1}, u_{2}\right)=\left\{\begin{array}{ll}0, & \text { if } u_{1}=0, \\ \infty, & \text { if } u_{1}>0 .\end{array}\right.$ and $f^{\left[x_{1}, x_{2}\right]}\left(u_{1}, u_{2}\right)=\left\{\begin{array}{ll}0, & \text { if } u_{1}=0, \\ \infty, & \text { if } u_{1}>0 .\end{array}\right.$.

In this case we obtain the following correct solution:

$$
\mathcal{I}\left(\left[x_{1}, x_{2}\right],\left[y_{1}, y_{2}\right]\right)= \begin{cases}{[1,1],} & \text { if } y_{1}=1, \\ {[0,0],} & \text { if } y_{1}<1 .\end{cases}
$$

Therefore, we have obtained 10 correct vertical sections in $\mathcal{L}^{I}$. Finally, we need to notice that it is not possible to find at least one solution $\mathcal{I}$ which is a fuzzy implication on $\mathcal{L}^{I}$ in the sense of Definition 3.7. The vertical sections 5), 6), 7) and 8) are not correct in this situation since we need to have

$$
\mathcal{I}\left(\left[x_{1}, x_{2}\right],[1,1]\right)=[1,1],
$$

for all $\left[x_{1}, x_{2}\right] \in L^{I}$. For all other possible solutions 1), 9), 11), 13), 15) and 16) we have

$$
\mathcal{I}([0,0],[0,0]) \neq[1,1],
$$

so it is not possible to find vertical solution, which is correct for $\left[x_{1}, x_{2}\right]=[0,0]$.

\section{Conclusion}

In this article we have discussed the following distributive equation

$$
\mathcal{I}\left(x, \mathcal{T}_{1}(y, z)\right)=\mathcal{T}_{2}(\mathcal{I}(x, y), \mathcal{I}(x, z)),
$$

when both t-norms are t-representable and such that $\mathcal{T}_{1}$ is generated from nilpotent t-norms, while $\mathcal{T}_{2}$ is generated from strict t-norms. In our future work we will concentrate on a dual situation, when $\mathcal{T}_{1}$ is generated from strict t-norms and $\mathcal{T}_{2}$ is generated from nilpotent t-norms.

\section{Acknowledgment}

This work has been supported by the Polish Ministry of Science and Higher Education Grant Nr N N519 384936.

\section{References}

[1] K.T. Atanassov, Intuitionistic fuzzy sets, VII ITKR's Session, Sofia deposed in Central Sci-Technical Library of Bulg. Acad. of Sci., 1697/84, 1983 (in Bulgarian).

[2] M. Baczyński, On a class of distributive fuzzy implications, Internat. J. Uncertain. Fuzziness Knowledge-Based Systems 9, 229-238 (2001)

[3] M. Baczyński, On the distributivity of fuzzy implications over continuous and Archimedean triangular conorms, Fuzzy Sets and Systems 161, 1406-1419 (2010)
[4] M. Baczyński, On the distributivity of implication operations over t-representable t-norms generated from strict t-norms in intervalvalued fuzzy sets theory, In: E. Hüllermeier, R. Kruse, F. Hoffmann (Eds.), Information Processing and Management of Uncertainty in Knowledge-Based Systems (IPMU 2010, Dortmund, Germany, June 28 - July 2, 2010. Proceedings, Part I), Communications in Computer and Information Science 80, Springer, Berlin Heidelberg, pp. 637-646 (2010)

[5] M. Baczyński, B. Jayaram, Fuzzy implications (Studies in Fuzziness and Soft Computing, Vol. 231), Springer, Berlin 2008.

[6] M. Baczyński, B. Jayaram, On the distributivity of fuzzy implications over nilpotent or strict triangular conorms, IEEE Trans. Fuzzy Systems 17, 590-603 (2009)

[7] J. Balasubramaniam, C.J.M Rao, On the distributivity of implication operators over $T$ - and $S$-norms, IEEE Trans. Fuzzy Systems 12, $194-$ 198 (2004)

[8] H. Bustince, M. Pagola, E. Barrenechea, Construction of fuzzy indices from fuzzy DIsubsethood measures: Application to the global comparison of images, Inform. Sci. 177, 906-929 (2007)

[9] W.E. Combs, J.E. Andrews, Combinatorial rule explosion eliminated by a fuzzy rule configuration, IEEE Trans. Fuzzy Systems 6, 1-11 (1998)

[10] W.E. Combs, Author's reply, IEEE Trans. Fuzzy Syst. 7, 371-373 (1999)

[11] W.E. Combs, Author's reply, IEEE Trans. Fuzzy Syst. 7, 477-478 (1999)

[12] B. De Baets, E. Kerre, M. Gupta, The fundamentals of fuzzy mathematical morphology, Part 1: Basic Concepts, Int. J. Gen. Syst. 23, 155-171 (1994)

[13] G. Deschrijver, E.E. Kerre, On the relationship between some extensions of fuzzy set theory, Fuzzy Sets and Systems 133, 227-235 (2003)

[14] G. Deschrijver, Ch. Cornelis, E.E. Kerre, On the representation of intuitionistic fuzzy t-norms and t-conorms, IEEE Trans. Fuzzy Systems 12, 45-61 (2004)

[15] G. Deschrijver, Ch. Cornelis, E.E. Kerre, Implication in intuitionistic and interval-valued fuzzy set theory: construction, classification and application, Internat. J. Approx. Reason. 35, 55-95 (2004)

[16] S. Dick, A. Kandel, Comments on "Combinatorial rule explosion eliminated by a fuzzy rule configuration", IEEE Trans. Fuzzy Syst. 7, 475-477 (1999)

[17] D. Dubois, S. Gottwald, P. Hajek, J. Kacprzyk, H. Prade, Terminological difficulties in fuzzy set theory - The case of "Intuitionistic Fuzzy Sets", Fuzzy Sets and Systems 156, 485-491 (2005) 
[18] J. Fodor, M. Roubens, Fuzzy preference modelling and multicriteria decision support, Kluwer, Dordrecht (1994)

[19] M.B. Gorzałczany, A method of inference in approximate reasoning based on intervalvalued fuzzy sets, Fuzzy Sets and Systems 21, 1-17 (1987)

[20] E.P. Klement, R. Mesiar, E. Pap, Triangular norms, Kluwer, Dordrecht (2000)

[21] M. Mas, M. Monserrat, J. Torrens, E. Trillas, A survey on fuzzy implication functions, IEEE Trans. Fuzzy Systems 15, 1107-1121 (2007)

[22] J.M. Mendel, Q. Liang, Comments on "Combinatorial rule explosion eliminated by a fuzzy rule configuration", IEEE Trans. Fuzzy Syst. 7, 369-371 (1999)

[23] D. Ruiz-Aguilera, J. Torrens, Distributivity of strong implications over conjunctive and disjunctive uninorms, Kybernetika 42, 319-336 (2005)

[24] D. Ruiz-Aguilera, J. Torrens, Distributivity of residual implications over conjunctive and disjunctive uninorms, Fuzzy Sets and Systems 158, 23-37 (2007)

[25] R. Sambuc, Fonctions $\Phi$-floues. Application á l'aide au diagnostic en patholologie thyroidienne, Ph.D. Thesis, Univ. Marseille, France, 1975

[26] E. Trillas, C. Alsina, On the law $[p \wedge q \rightarrow r]=$ $[(p \rightarrow r) \vee(q \rightarrow r)]$ in fuzzy logic, IEEE Trans. Fuzzy Systems 10, 84-88 (2002) 